\title{
Parametric integration of design features selection with recording of construction of working machines
}

\author{
Piotr Gendarz ${ }^{*}$ \\ Institute of Engineering Processes Automation and Integrated Manufacturing Systems, \\ Silesian University of Technology, Konarskiego Street 18a, 44-100 Gliwice, Poland
}

\begin{abstract}
Adaptation of the graphic program for constructing of a specific class of technical means, being the specialty of the design and construction office, is the basic challenge of the market economy. This office that prepares the offer and then the competitive construction of the technical means in the shortest possible time as a result obtains the order. This effect is enabled by graphic software applications.
\end{abstract}

\section{Introduction}

Creating ordered construction families of working machines, requires an integral look at the process of selecting design features as well as at construction recording, taking into account: serial production processes, use, and their recirculation and disposal. The basic forms of ordered families of structures are: series of types and modular systems of construction of working machines.

The creation of ordered construction families of working machines is a very laborious and tedious issue $[1,2]$. Therefore, it is justified to develop methods of computer aiding of the stages of this process. The most labour-consuming and at the same time susceptible to computer aiding is the selection of values of dimensions in which the $\gamma$ assignment $[2,3]$ is implemented. On the basis of the developed methodology, some methods for determining the quantitative value of constructional features were developed [2]: traditional method, construction similarity method, algorithmic method, neural network method.

The subject of considerations in this publication is the algorithmic method. It is a method of assigning, using the logic operators, dimensions of the components to unified parameters of a technical means. These dimensions are described a parametric way. And the components have already defined their constructional form. The integration of such selected design features with the design record is carried out with the use of various parameterization methods used in graphic programs (term describing the group of CAD programs and other applications resulted in a form of a technical drawing).

Practical verification of the integration of methods of selection and parametric recording was carried out on the example of families of hydraulic cylinders used in: construction machinery, mining equipment of the extractive industry (supports, heading equipment,

\footnotetext{
* Corresponding author: piotr.gendarz@polsl.pl
} 
shearing machines) and metallurgical slag trucks and belt conveyors. As the result of the typification, the design diversity of hydraulic cylinder assemblies is presented in Fig. 1.

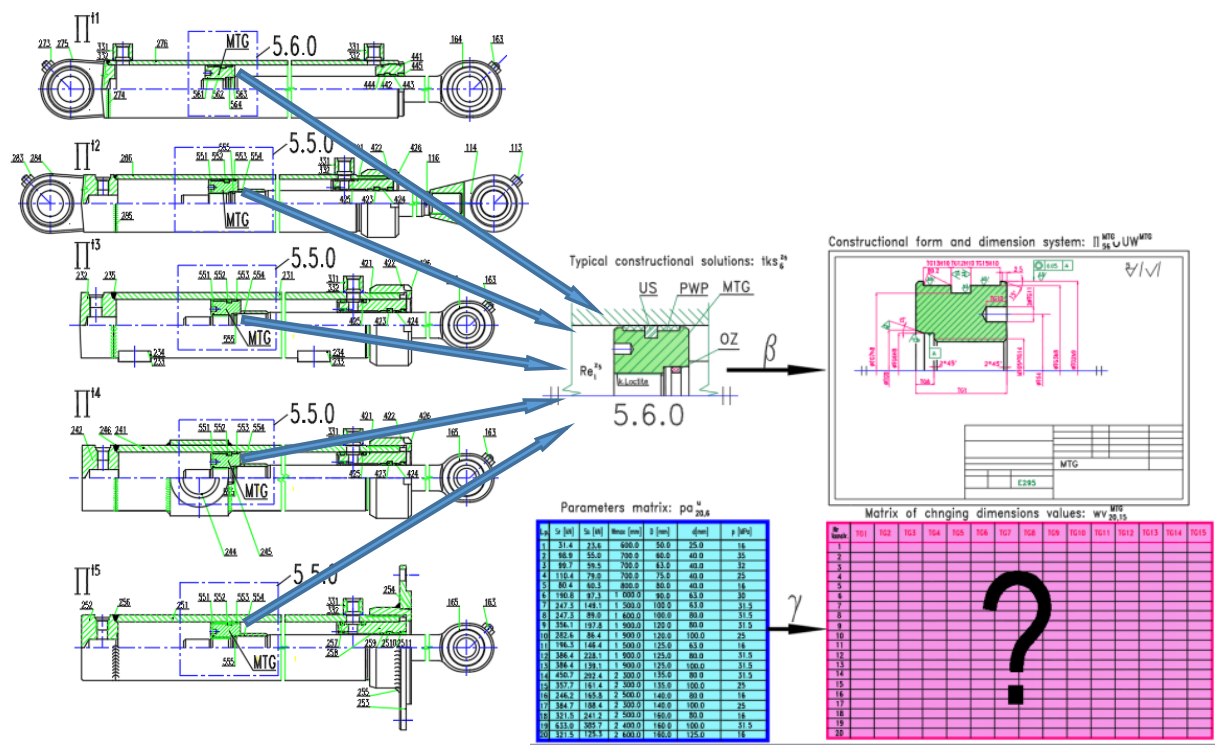

Fig. 1. Typical design forms of design solutions for hydraulic cylinders with the creation of the $\gamma$ assignment for MTG design modules

The following types of assignments were distinguished in the process:

$\alpha$ - between unified characteristic features and typical constructional solutions,

$\boldsymbol{\beta}$ - between typical constructional solutions and typical constructional forms of elements,

$\gamma$ - between unified characteristic features and dimension values,

$\boldsymbol{\delta}$ - between unified characteristic features and optimally differentiated dimension values, which mainly affects the number of ordered components of the construction family.

\section{Assignment $\gamma$ - selection of dimension values}

The algorithmic method in the ordered family of constructions is the method of creating the assignment $\gamma$ between the unified values of parameters $x_{i a}^{u}$ and quantitative features of the construction of elements $y_{m l}^{e j} ;\left(l=1, l v_{j}\right)$, in the order and relation created on the basis of the graph of the coupling relations $G\left\langle\pi_{r w}^{t e j}\right\rangle$ using operators [O], according to the dependence below [3]:

$$
y_{m l}^{e j}(j=1, j z)=[O] \cdot x_{i a}^{u}, \text { for } m=i, \text { dla } \mathrm{m}=\mathrm{i} .
$$

The model of assignment creation is shown in Fig. 2. The basis for selection the quantitative constructional features are operators [O]. The operators determine relations between values of unknown dimensions $y_{m, n z}^{e j}$ based on values of parameters $x_{i a}^{u}$ or values of known dimensions $y_{m, z n}^{e j}$ :

$$
x_{i a}^{u} \stackrel{[0]}{\rightarrow} y_{m, n z}^{e j} \text { or } y_{m, z n}^{e j} \stackrel{[0]}{\rightarrow} y_{m, n z}^{e j}
$$

The following types of operators have been distinguished (see also Fig.3):

- geometric operators $\mathrm{O}_{\mathrm{G}}$, 
- $\quad$ strengths operators $\mathrm{O}_{\mathrm{W}}$,

- $\quad$ selected elements operators dobieranych $\mathrm{O}_{\mathrm{D}}$,

- manufacturing process operators $\mathrm{O}_{\mathrm{P}}$,

- constructional similarity operators $\mathrm{O}_{\mathrm{C}}$,

- conjugated dimensions operators $\mathrm{O}_{\mathrm{s}}$.

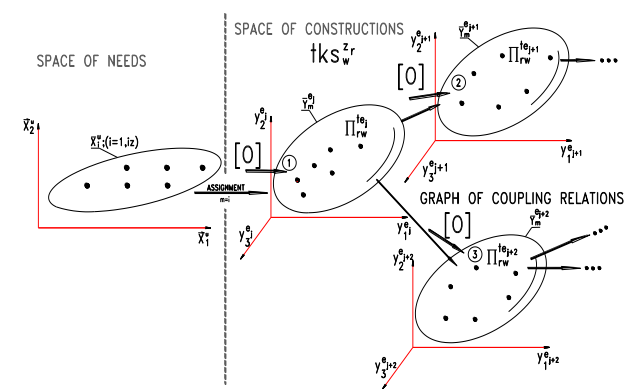

Fig. 2. Model of algorithmic creation of the assignment $\gamma$

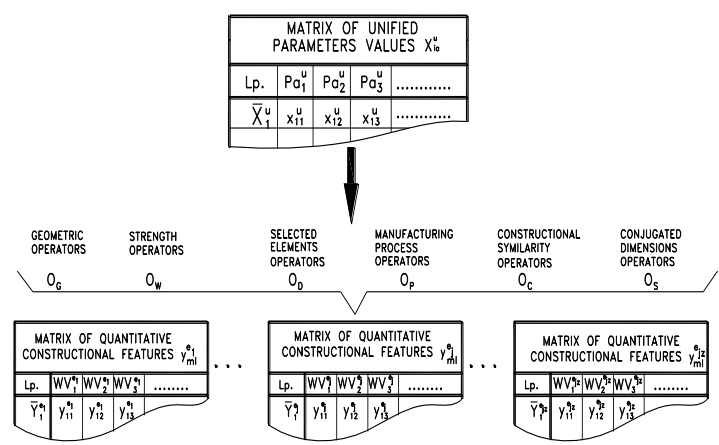

Fig. 3. Operators of selection the quantitative constructional features

Values of unknown dimensions $y_{m, n z}^{e j}$ are usually determined using the geometric operators . This group of operators includes such operators like: arithmetic, trigonometric, conditional and adjusting values to the series of normal numbers:

$$
O_{G} \in O_{A R} \cup O_{T R} \cup O_{W A} \cup O_{N O}
$$

Strength operators are used to introduce the conditions of strength verification of the construction being analyzed. Because these conditions are dependent on the dimensions being chosen, this is why they can be used as the basis for determining the values of unknown dimensions $y_{m, n z}^{e j}$ :

$$
\begin{aligned}
& \sigma=f\left(y_{m, n z}^{e j}\right) \leq k_{\sigma} \\
& \tau=f\left(y_{m, n z}^{e j}\right) \leq k_{\tau} \stackrel{o_{w}}{\rightarrow} y_{m, n z}^{e j} \\
& p=f\left(y_{m, n z}^{e j}\right) \leq k_{p}
\end{aligned}
$$

In the process of determining the values of dimensions $y_{m, n z}^{e j}$, the selected elements operators (catalog elements, normalized ones, manufactured by cooperators) are used as the first. In this group of operators one should first use the criterion of selecting the typical values by defining the parameters of the selected element $\bar{X}_{j}^{D}$ on the basis of known dimensions 
$y_{m, z n}^{e j}$, and then applied the criteria of the assembly, to choose the values of dimensions of their arrangement $w_{z a b}^{D}$. The general model of the application of selected elements operators is determined by:

$$
\begin{aligned}
& y_{m, z n}^{e j} \stackrel{o_{D}}{\rightarrow} \bar{X}_{j}^{D} \\
& \bar{X}_{j}^{D} \stackrel{O_{D}}{\rightarrow} w_{z a b}^{D} \\
& y_{m, n z}^{e j}=f\left(w_{z a b}^{D}\right)
\end{aligned}
$$

Fulfilling the criteria resulting from the right of manufacturing possibilities, the manufacturing process operators are designated. These operators are used similarly to the operators of standardized elements :

$$
\begin{aligned}
& y_{m, z n}^{e j} \stackrel{o_{P}}{\rightarrow} \bar{X}_{j}^{P} \\
& \bar{X}_{j}^{P} \stackrel{O_{P}}{\rightarrow} w_{c}^{P} \\
& y_{m, n z}^{e j}=w_{c}^{P}
\end{aligned}
$$

The constructional similarity operators can be interpreted as follows: if for the given parameter values $\bar{X}_{0}^{u}$ the values of the dimensions $\bar{Y}_{0, z n}^{e j}\left(y_{o, z n}^{e j}\right)$ are known, then maintaining the condition of construction congruency $[4,5]$, it is possible to determine which dimension values will be accepted by the newly constructed element $\bar{Y}_{m, n z}^{e j}\left(y_{m, n z}^{e j}\right)$ for new vectors of needs $\bar{X}_{i}^{u}$ :

$$
\begin{aligned}
\bar{X}_{0}^{u} & \rightarrow \bar{Y}_{0}^{e j}\left(y_{0, z n}^{e j}\right) \\
\bar{X}_{i}^{u} & \rightarrow \bar{Y}_{m}^{e j}\left(y_{m, z n}^{e j}\right)
\end{aligned}
$$

The basis for creating conjugated dimensions operators is the graph of coupling relations $G\left(\pi_{r w}^{t e j}\right)$, Fig. 4a [5]. On the basis of operators of conjugated dimensions $\mathrm{O}_{\mathrm{S}}$ and known dimensions $y_{m, z n}^{e j}$ the values of unknown dimensions of the cooperating element $y_{m, n z}^{e j+1}$ are determined:

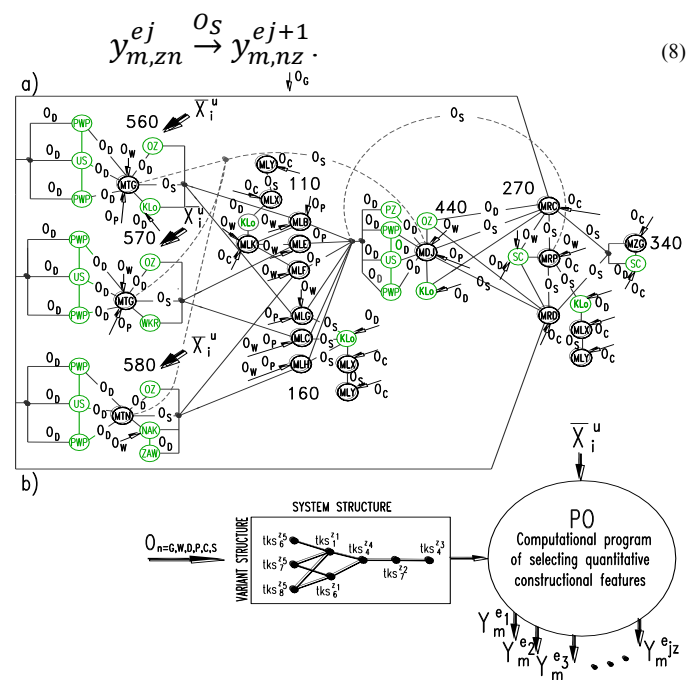

Fig. 4. Model of creating the computer program designating the assignment $\gamma$

Presented formalized operations allow determining the values of particular features of the analyzed type of a construction. 
The graph of the coupling relations $G\left(\pi_{r w}^{t e j}\right)$ and all of the mentioned operators are the basis for creating the algorithm for choosing the values of dimensions. This algorithm is based on the system and variant structure of the construction family [3], what is seen in Fig. 4b. The final result is the computer program elaborated in Visual Fortran, $\mathrm{C}++$, AutoLISP or another programming language that allows calculating the values of dimensions of the components of the construction family $[2,3]$ for the unified values of parameters, what is presented in Fig. 5.

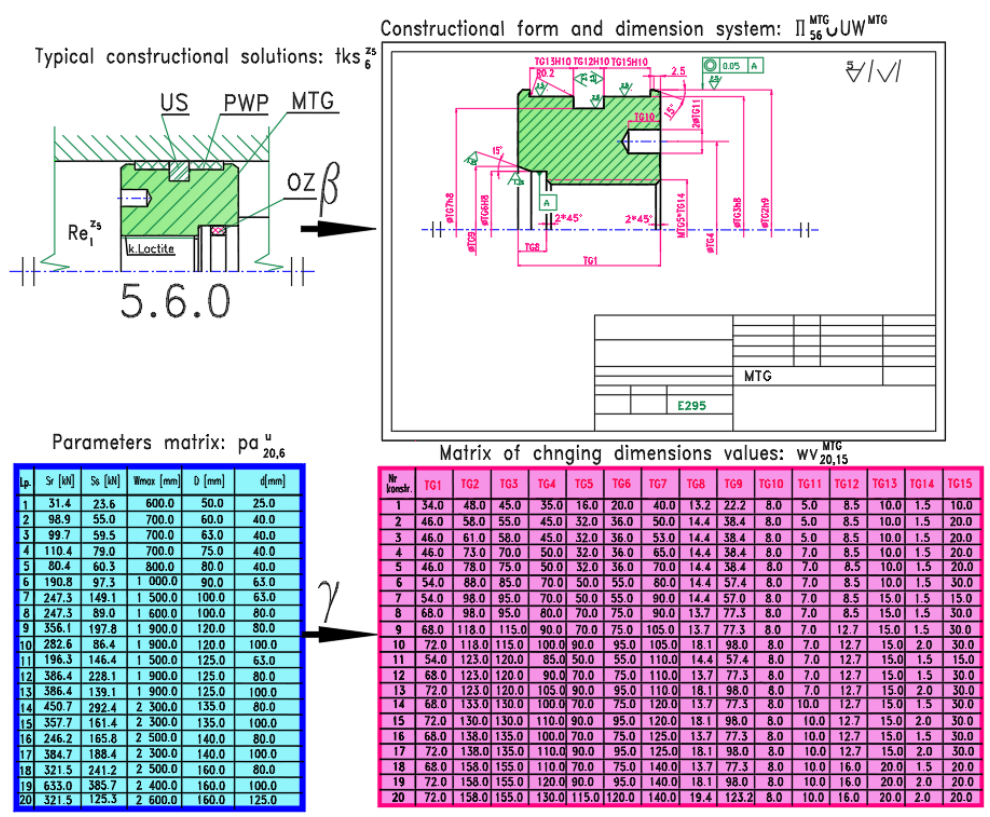

Fig. 5. Assignment $\gamma$

Constructional modules are created only after optimizing the variety of dimension values [3]. The calculation program PO contains a variant structure, distinguishing the sub-programs of the variant $P O_{w}^{z r}$. These sub-programs for the variants are divided next into program modules $P O_{r w}^{e j}$ that correspond to typical constructional forms of elements $\pi_{r w}^{t e j}$. The structure of the program is additionally extended with program modules: of the strength verification (containing operators $\mathrm{O}_{\mathrm{W}}$ ), of selected elements choosing (containing operators $\mathrm{O}_{\mathrm{D}}$ ), including the technological process parameters (including operators $\mathrm{O}_{\mathrm{D}}$ ), of constructional similarity (containing operators $\mathrm{O}_{\mathrm{C}}$ ), and for results saving.

\section{Parameterization}

Parameterization is understood as a form of a construction description in which the constructional form and the system of dimensions are mapped in the computer's operating memory (computer program, 2D models, 3D models), while variable values of dimensions are read from external bases. In this way, a combination of the qualitative constructional features (geometric constructional form) with the quantitative constructional features (dimension values) takes place. Changing the dimension values in the parametric description of a construction is associated with a change of the model of the created element description. Its results in a construction description which is presented with high accuracy in the appropriate scale. 
Depending on the method of introducing quantitative constructional features, next parameterization types are distinguished $[2,6]$ :

- dynamic,

- relational,

- graphic,

- program.

Dynamic parameterization consists in a manual change of the values of selected dimensions, as a result of which the construction description is dynamically modified to the given dimension values. It is rarely used in the description of series of types of constructions.

Relational parametrization allows changing the description of a construction by changing the values of dimensions determined in a relational way to an independent variable, e.g.: the technical means parameter, a significant dimension. The relations between the independent variable and the dimension values are stored in the appropriate editor using the algebraic, trigonometric and logical relations. So it is possible to elaborate algorithm including mathematical relations which could be easy transformed into any computer program.

When the specified dimension is subordinated to the independent variable, the dimensional values are calculated, and then the existing construction description is updated. An example of relational parameterization can be the parameterization of the cylinder sleeve mounted on the rocker arms in the advanced graphic program I-DEAS. Relationships between dimensions are saved in the editor designed for relations establishing, Fig. 6.

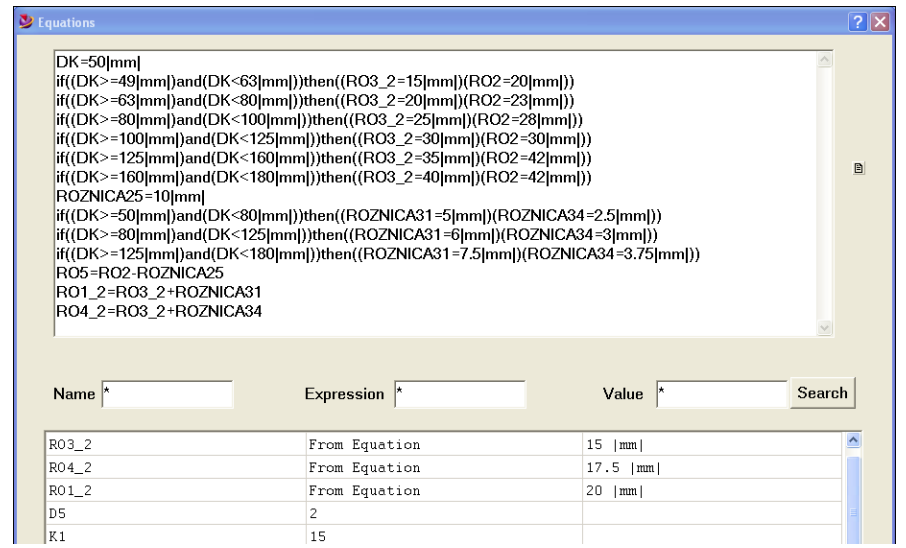

Fig. 6. Editor designed for relations establishing Fig. 7.

On the basis of the relational parameterization, 3D models of elements could be created,

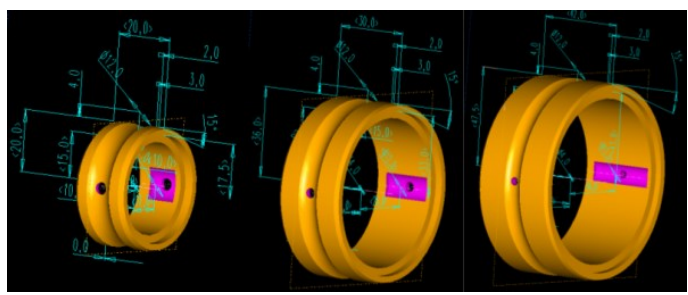

Fig. 7. Models of MRO (code of elements) for the piston diameter $\mathrm{DK}=50, \mathrm{DK}=100, \mathrm{DK}=160$

Graphical parameterization does not require programming skills, but only the possibilities of a graphic program are used. The input data in this form of parameterization are tables of variable dimension values, Fig. 8 , adapted to the created parametric description. 


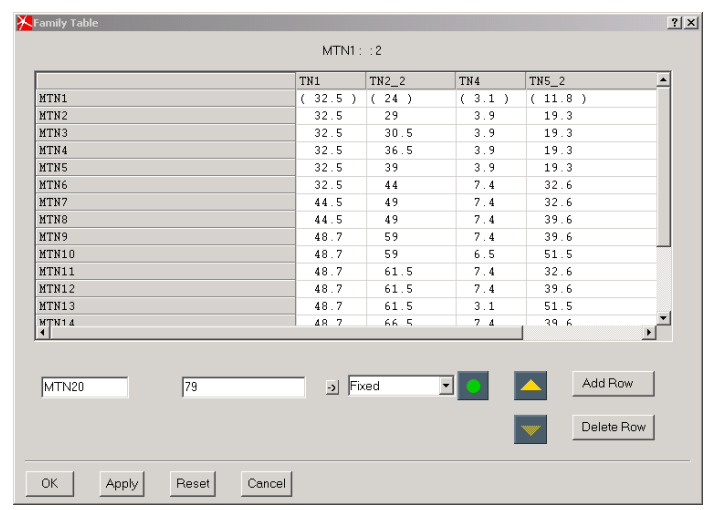

Fig. 8. Structure of a table with variable dimensions

Among the parameterization methods, mentioned above, the program parameterization is used the most often. This method is described in detail in the next chapter.

\section{Integration of constructional features selection with the construction description using program parameterization}

Program parameterization consists in utilizing the programming language to create the description of a construction in a graphic program. It is a form of parameterization that allows integrating the process of selection of constructional features with the construction description.

An example of such integration can be the use of special languages dedicated for graphic programs. The most often used are:

- programming language GRIP in the advanced graphic environment PLM Siemens NX,

- programming language AutoLISP in the graphic program AutoCAD Mechanical.

As the example of the program parameterization is presented the application of its second form related with the creation of $2 \mathrm{D}$ parametric descriptions. The programming language AutoLISP, like any other programming languages, uses such operations like: data reading, calculations performing according to complex mathematical relations, conditional loops functions, iterative loops ones, conditional "if" ... "to" ... functions, data outputting in the form of arrays (charts). In addition, it uses the commands of the graphic program, which allows selecting the constructional features and integrating them with the description of a construction.

The parametric description in this form of parameterization is represented by a calculation program saved in the AutoLISP language, intended for describing the construction of elements, assemblies or repetitive components such as drawing labels, surface roughness marks, collective surface roughness ones, shape and position tolerances symbols. A particular application of program parameterization is the description of series of types of construction based on calculation algorithms or data tables.

There are two basic ways to integrate the selection of constructional features with the description of a construction:

- direct,

- indirect.

Direct integration involves the use of the AutoLISP program and direct determination of the dimension values in the parametric program based on the values of the characteristic 
features. The calculation program, along with the commands of construction description, may be in the form of a *.LSP file, which is run from the graphical program level by the LOAD command or by a function run from the level of any parametric program.

For more complex calculations, indirect integration is used. It involves the use of external computational programs written in a programming language, e.g. C, Visual Fortran, Visual Basic, and the results of calculations are gathered in properly formatted tables. Initiating the program of the *.EXE or *.COM type can be implemented by initiating the AutoLISP program from any parametric program or from menu using the instruction as follow: (command "..start" (strcat kxx4 "OBLICZENIA")), where "OBLICZENIA" is the name of the program of the EXE type located in an available catalog. In turn, the created parametric programs *.LSP allow reading the created tables and using the commands of the graphic program they create description of a construction. This connection of calculations with the construction description is one of the possibilities of creating flexible systems of modular constructions.

In advanced graphic programs, the documentation of the $2 \mathrm{D}$ constructions description is still dominant in the form of a traditional construction drawings, despite numerous attempts to create a $3 \mathrm{D}$ construction descriptions with the system of dimensions and a description of other constructional features, Fig. 9.

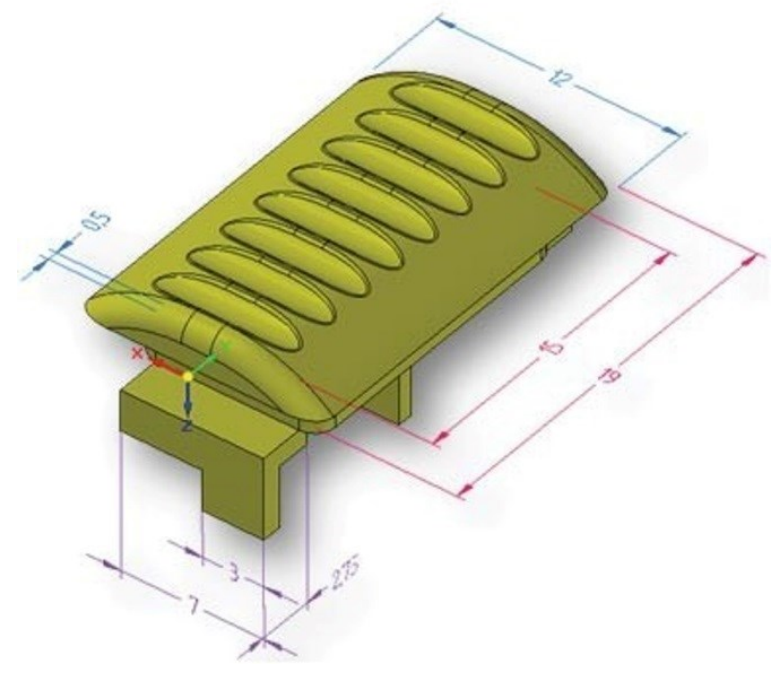

Fig. 9. 3D Description of a construction in a form of a working 3D drawing [7]

For more complex elements, this description (Fig. 9) becomes less readable, therefore still dominant is the 2D description of a construction, created semi-automatically from the 3D model in the Drafting module of a graphic software, what is presented in Fig. 10. In order to omit the labor-intensive transition from 3D description to 2D one, a direct description of the $2 \mathrm{D}$ construction is created, e.g. using program parameterization $[2,6]$.

During creating the parametric program of the construction description, in the form of a working drawing, one could distinguish auxiliary and main operations coded in the AutoLISP language. Auxiliary operations are mainly: cleaning the screen from previous records, reading from the tables or making a selection of constructional features, determining the format and scale of the drawing, adjusting the values of dimensions to the scale, creating the frame and drawing label. The main operations include the description of: a constructional form, hatching of the cross-sections, drawing the axis of symmetry, creating the system of 
dimensions, the marks of surface roughness, shape and position tolerances, as well as additional information and marks, and writing variable data in the label.
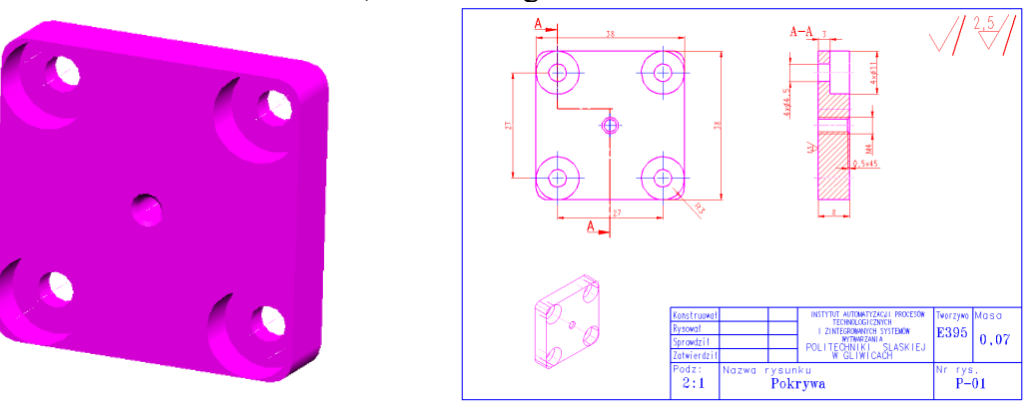

Fig. 10. Transition from the $3 D$ description of a construction to the $2 D$ one

For example, the description of a constructional form is carried out point by point, where the coordinates of the characteristic points of the outline are determined as a function of dimensional values:

$$
\left\langle x_{n}, y_{n}\right\rangle=f\left(y_{i l}^{t e j}\right)
$$

An example of such creating of the parametric program is shown in Fig. 11. Fig. 11a presents the construction with an open system of dimensions. In contrast, in Fig. 11b are shown the characteristic points of the outline of the element whose coordinates are to be calculated on the basis of dimensional values. A fragmentary description of the parametric program in the AutoLISP programming language is shown in Fig. 11c. The exemplary construction descriptions based on this parametric program and created in the graphic program are shown on the right side of Fig. 11. They represent an entire 2D documentation according technical requirements.

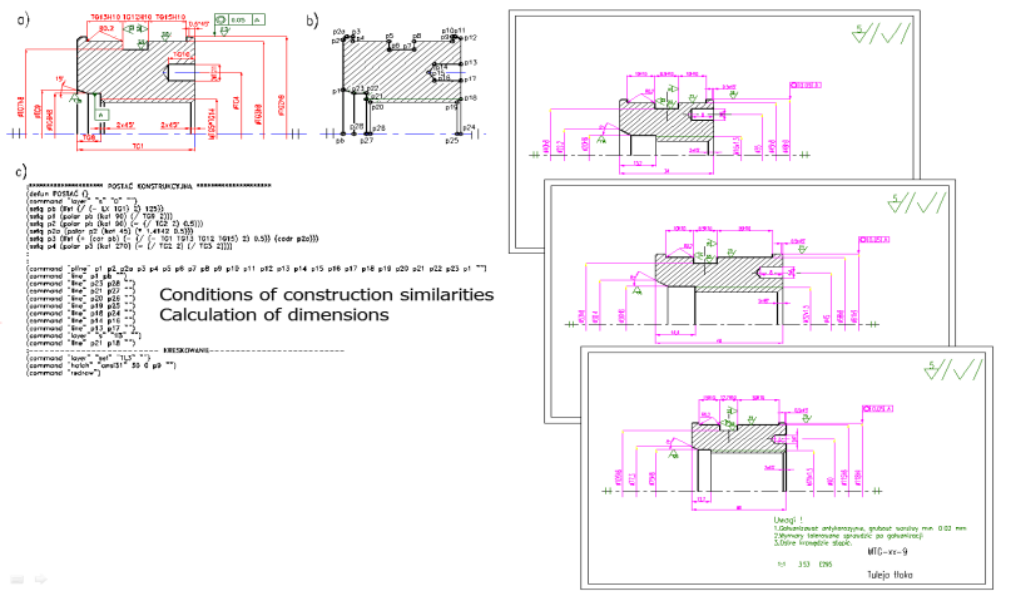

Fig. 11. Stages of creating the parametric program of constructional form description of an element

Parametric programs could be saved using a special Visual LISP editor of the graphics program AutoCAD Mechanical. It gives the possibility for more efficient programming, among others in terms of: verification of the number of open and closed brackets, quick access and use of AutoLISP commands as well as compilation of this program. Parametric programs, created in AutoCAD Mechanical, work very effectively and quickly. A certain additional possibility is also the utilization of dialog windows. The dialog windows are program created using the DCL programming language which is initiated via the AutoLISP program command editor. 


\section{Summary and conclusions}

The creation of modern, more sophisticate graphic software applications facilitates the creation of description of repeating parts of technical drawings, e.g. axes of symmetry, collective mark of surface roughness, frames as well as labels. It is also possible to create more complicated descriptions of construction of ordered families of construction in the form of constructional documentation - assembly drawing, working drawings, and bills of elements. In addition, one can elaborate the repeating stages of the design and construction process, such as the selection of catalog and standardized elements, optimization of joints due to costs analysis as well as optimization of sheet cutting in the given format. Therefore, basing on the methods presented in the paper, it will soon be possible to automate some of the design procedures to eliminate human involvement in them. The next step may be to include elements of artificial intelligence in the indicated procedures.

Graphic programs applications increase the competitiveness of design and construction offices. But their rational utilization requires not only the skills of designing, constructing, describing of a construction, but also programming ones related with the AutoLISP language for creating parametric programs, the DISEL language for creating customized menu, the DCL language for creating: dialog windows, blocks, blocks with attributes and slides. It is also required to learn external computational programs in programming languages such as Visual Fortran, C, Visual Basic. Therefore, it seems important that design offices are represented by an increasingly diverse profession.

\section{References}

1. A. Gwiazda, A. Sekala, W. Banas, S. Topolska, K. Foit, Z.Monica, IOP Conference Series-Materials Science and Engineering (2017), Volume 227, s. 1-7

2. P. Gendarz, The parametric record ordered families of machine design. Monograph, Gliwice (2010)

3. P. Gendarz, Applications of graphic programs in structured construction families. Monograph, Gliwice (1998)

4. P. Gendarz, Forschung Im Ingenieurwesen - Engineering Research, Springer 73(4):245-255, (2009)

5. P. Gendarz, Forschung Im Ingenieurwesen - Engineering Research, Springer 74:175184 (2010)

6. P. Gendarz, Forschung Im Ingenieurwesen - Engineering Research, Springer 77(3):105-115 (2010)

7. Documentation for Siemens NX v. 8.5. 